American Journal of Applied Sciences 1 (3): 209-214, 2004

ISSN 1546-9239

(C) Science Publications, 2004

\title{
Depth Estimation of 2-D Magnetic Anomalous Sources by Using Euler Deconvolution Method
}

\author{
${ }^{1,3}$ M.G. El Dawi, ${ }^{1}$ Liu Tianyou, ${ }^{1}$ Shi Hui and ${ }^{2}$ Luo Dapeng \\ ${ }^{1}$ Faculty of Geophysics and Space Information, \\ China University of Geosciences, Wuhan, 430074, China \\ ${ }^{2}$ Faculty of Electronic Engineering, \\ China University of Geosciences, Wuhan, 430074, China \\ ${ }^{3}$ School of Earth Sciences, Faculty of Science and Technology, \\ Al Neelain University, Khartoum, Sudan
}

\begin{abstract}
Euler deconvolution method is the method of depth estimation which is best suited for anomalies caused by isolating and multiple anomalous sources. In the present study, algorithmic programs based on generalizing linear inverse theory, uses a least square sense to solve for Euler's equation is being written. The Euler technique that can estimate the location of a simple body from measurements of the magnetic field could be applied to a long profile of measurements, by dividing the profile into the windows of consecutive measurements, each window providing a single estimate of depth and source location. Acceptable solutions for features of interest may involve some trial and error by adjusting the structural index and the window size. When all such measurements are plotted they tend to cluster around magnetization of geologic interest. Some indication of the source type can be gained by varying the structural index for any particular feature. Shallow features can be deconvolved well by using small window to reduce source interference. By using $n=1$ and $n=1.5$, and deconvolved with small window sizes, our program yields good tight clustering and acceptable depths of the anomalous igneous body located along aeromagnetic profile in the south of Amenzi area, Inner Mongolia, North China. The depths obtained by using the structural index of $n=1.5$ varying between 700-900 $\mathrm{m}$. The method yielded useful solutions with an acceptable depth estimate.
\end{abstract}

Key words: Euler Deconvolution, Structural Index, Window Size, Amenzi Area

\section{INTRODUCTION}

The Euler deconvolution method is used for rapid interpretation of potential field (magnetic and gravity) data. It is particularly good at delineating contacts and rapid depth estimation. This technique belongs to automatic depth estimate methods and, is designed to provide computer-assisted analysis of large volumes of magnetic and gravity data.

Euler's equation has been used by a number of authors for analyzing both magnetic anomalies ${ }^{[1,2,3]}$ and gravity anomalies ${ }^{[4]}$. Euler's homogeneity relationship offers a quasi-automated method to derive the plan location and depth estimation of buried Ferro-metallic objects from magnetic data. Euler's homogeneity equation relates the magnetic field and its gradient components to the location of the source with the degree of homogeneity expressed as a structured index, ${ }^{[1]} .{ }^{[1]}$ Developed the technique and applied it to profile data. ${ }^{[3]}$ Developed the technique most widely used version for grid-based data. Also recent improvements on the technique had occurred which included the estimation of the structural index ${ }^{[5]}$. ${ }^{[6]}$ Developed a multiple-source generalization of Euler deconvolution, which is capable of handling complex systems that the single-source algorithm can only deal with approximately.

The advantages of this technique over more conventional depth interpretation methods (i.e. Characteristic curves, inverse curve matching), are that no particular geological model is assumed, and that the deconvolution can be directly applied and interpreted even when particular model, such as a prism or dyke cannot properly represent the geology.

Theory of euler equation: However, for applying the Euler's expression to profile or line-oriented data (2-D sources). In this case, the x-coordinate is a measure of the distance along the profile, and the y-coordinate can be set to zero along the entire profile:

$\left(\mathrm{x}-\mathrm{x}_{0}\right) \frac{\partial \Delta \mathrm{T}}{\partial \mathrm{x}}-\mathrm{z}_{0} \frac{\partial \Delta \mathrm{T}}{\partial \mathrm{z}}=-\mathrm{n} \Delta \mathrm{T}(\mathrm{x})$

Rearrangement of the above expression yields:

$x_{0} \frac{\partial \Delta T}{\partial x}+z_{0} \frac{\partial \Delta T}{\partial z}=x \frac{\partial \Delta T}{\partial x}+n \Delta T(x)$ 
where $\left(\mathrm{x}_{0}, \mathrm{z}_{0}\right)$ is the position of a 2-D magnetic source whose total field $\mathrm{T}$ is detected on $(\mathrm{x}, \mathrm{z})$. The total field has a regional value of $\mathrm{B}$, and $\mathrm{n}$ is a measure of the falloff rate of the magnetic field. $\mathrm{N}$ is directly related to the source shape and is referred to as the structural index ${ }^{[1]}$. By evaluating the total field $\mathrm{T}$ and its derivatives (calculated or measured, The gradient can be calculated using standard potential theory in the space or wave number domain or the vertical gradient may be calculated and can be used directly in the above equation) at all points on a magnetic data set, a system of simultaneous linear equations is obtained with the unknowns being the $\mathrm{x}_{0}, \mathrm{z}_{0}$ represent the location and depth of the magnetic source, $n$, the structural index of the source and $\mathrm{B}$ the regional magnetic field. Using the derivatives of potential-field anomalies enhance the field associated with shallow features and de-emphasize the field from deeper sources. Used appropriately, the method is suitable for characterizing sources of all potential-field data and/or their derivatives, as long as the data can be regarded mathematically as continuous ${ }^{[7]}$.

The application of equation (2) directly to the observed data makes the exact solution unreliable and erratic. The problem of removing the bias from the observed data is solved in the following way by ${ }^{[1]}$.

Assume the anomalous field is perturbed by a constant amount B in the window in which equation (2) is being evaluated. The observed quantity is:

$\mathrm{T}(\mathrm{x})=\Delta \mathrm{T}(\mathrm{x})+\mathrm{B}$

where, $\mathrm{B}$ is a constant in the coordinate $\mathrm{x}$ over the portion of the profile where the analysis is being made. Solving equation (3) for $\Delta \mathrm{T}$, substituting into equation (2) and arranging terms yield:

$x_{0} \frac{\partial T}{\partial x}+z_{0} \frac{\partial T}{\partial z}+N B=x \frac{\partial T}{\partial x}+n T$

Equation (4) with three unknowns $\mathrm{x}_{0}, \mathrm{z}_{0}$, and $\mathrm{B}$ can be solved by using a least squares procedure.

The proposed method involves setting an appropriate value of the structural index of the suspected source bodies and then solving the system by least-squares inversion for an optimum $\mathrm{x}_{0}, \mathrm{z}_{0}$ and $\mathrm{B}$. The inversion process also yields an uncertainty (standard deviation) for each of the fitted parameters. These uncertainties may be used as criteria for acceptance or rejection of solutions.

To solve for the source location $\mathrm{x}_{0}, \mathrm{z}_{0}$ the process normally solving the following least square normal equation associated with equation (4):

$\mathrm{G}^{\mathrm{T}} \mathrm{Gm}=\mathrm{G}^{\mathrm{T}} \mathrm{d}$

$\mathrm{m}=\mathrm{G}^{\mathrm{T}} \mathrm{d}\left(\mathrm{GG}^{\mathrm{T}}\right)^{-1}$ where, $\mathrm{m}$ is the vector of unknown parameters:

$$
\begin{gathered}
m=\left[\begin{array}{l}
X_{0} \\
Z_{0}
\end{array}\right] \\
G^{T} G=\left[\begin{array}{l}
\left\langle\frac{\partial T}{\partial x}, \frac{\partial T}{\partial x}\right\rangle\left\langle\frac{\partial T}{\partial x}, \frac{\partial T}{\partial z}\right\rangle \\
\left\langle\frac{\partial T}{\partial x}, \frac{\partial T}{\partial z}\right\rangle\left\langle\frac{\partial T}{\partial z}, \frac{\partial T}{\partial z}\right\rangle
\end{array}\right]
\end{gathered}
$$

And:

$\mathrm{G}^{\mathrm{T}} \mathrm{d}=\left[\begin{array}{l}\left\langle\frac{\partial \mathrm{T}}{\partial \mathrm{x}}, \mathrm{x} \frac{\partial \mathrm{T}}{\partial \mathrm{x}}\right\rangle+\left\langle\frac{\partial \mathrm{T}}{\partial \mathrm{x}}, \mathrm{z} \frac{\partial \mathrm{T}}{\partial \mathrm{z}}\right\rangle+\mathrm{n}\left\langle\frac{\partial \mathrm{T}}{\partial \mathrm{x}}, \mathrm{T}\right\rangle \\ \left\langle\frac{\partial \mathrm{T}}{\partial \mathrm{z}}, \mathrm{x} \frac{\partial \mathrm{T}}{\partial \mathrm{x}}\right\rangle+\left\langle\frac{\partial \mathrm{T}}{\partial \mathrm{z}}, \mathrm{z} \frac{\partial \mathrm{T}}{\partial \mathrm{z}}\right\rangle+\mathrm{n}\left\langle\frac{\partial \mathrm{T}}{\partial \mathrm{z}}, \mathrm{T}\right\rangle\end{array}\right]$

Steps of processing: The Following Flow Chart Shows the Input and Steps of Processing:

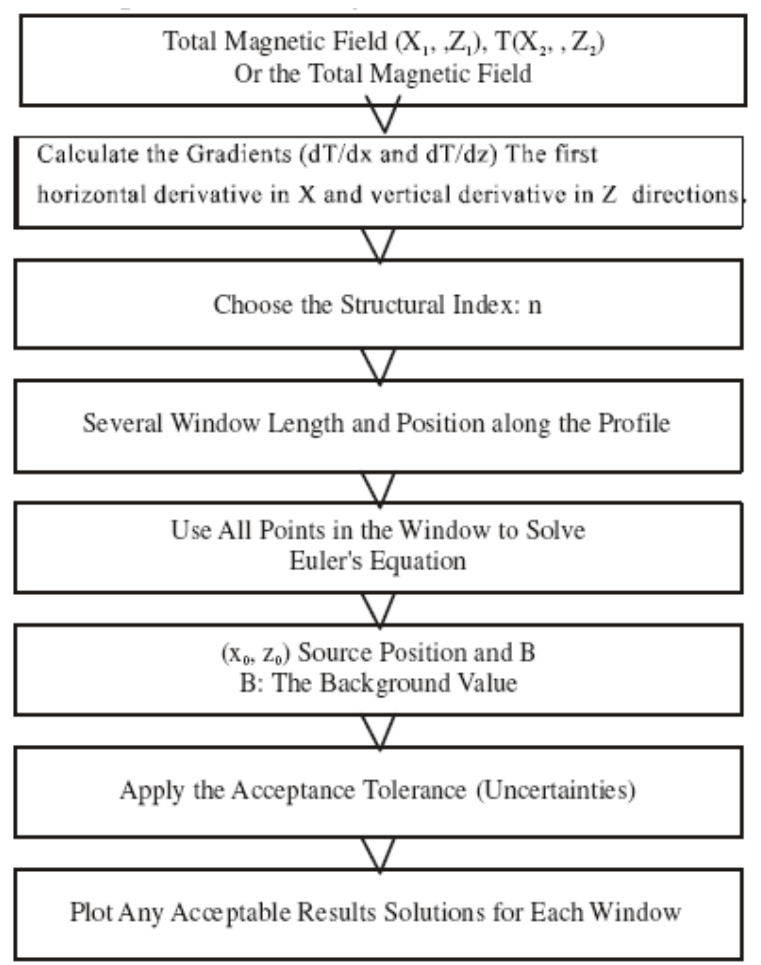

Interpretation: Using MATLAB software version 6.5 for programming, a program uses the generalized linear inverse method in a least square sense to solve the Euler's equation has been written. It will be shown that the general inverse can be constructed so as to get a better fit to the data (in a least square sense) become stabilized, so that it is less critical to choose a large number of model parameters than with the ordinary least square technique. 
To discuss our model construction, from the linear equation;

$$
\mathrm{G}_{\mathrm{M} \times \mathrm{N}} \mathrm{m}_{\mathrm{N} \times 1}=\mathrm{d}_{\mathrm{M} \times 1}
$$

Where:

$\mathrm{M}=$ The number of observations

$\mathrm{N}=$ The number of model parameters

The least square solution to linear problems, objective function:

$$
E=\left(d-G_{m}\right)\left(d-G_{m}\right)^{T}
$$

$=d d^{T}-G m d^{T}-d m^{T} G^{T}+G m m^{T} G^{T}$

Let $\frac{\partial \mathrm{E}}{\partial \mathrm{m}^{\mathrm{T}}}=0$, then we have:

$$
-d^{\mathrm{T}} \mathrm{G}+\mathrm{Gm}^{\mathrm{T}} \mathrm{G}^{\mathrm{T}}=0
$$

$m=\left(G^{T} G\right)^{-1} G^{T} d$

This is what is called the normal equation:

$$
\mathrm{m}=\mathrm{G}_{\mathrm{L}} \mathrm{d}
$$

If $M>N=r$, where $r=\operatorname{rank}$ of matrix $G$, an overdetermined system, then some constrains must be added to effect more information (M-N) from the observation. This is what is called L. S solution. So that from equation (6):

$\mathrm{m}=\mathrm{G}^{\mathrm{T}}\left(\mathrm{GG}^{\mathrm{T}}\right)^{-1} \mathrm{~d}$

The above equation is called the generalized inversion.

If $\mathrm{G}$ is $\mathrm{M} \times \mathrm{N}$ matrix which is singular matrix. The matrix $\mathrm{G}$ can be factored as.

By applying the matrix decomposition theory of ${ }^{[8]}$.

$\mathrm{G}=\mathrm{U}_{\mathrm{r}} \wedge_{\mathrm{r}} \mathrm{V}_{\mathrm{r}}^{\mathrm{T}}$

The above equation is called Singular Value Decomposition (SVD) of singular matrix $G, \wedge_{r}$ is a diagonal matrix with $\mathrm{r}$ singular values on its main diagonal and zero elsewhere:

$$
\begin{aligned}
& \wedge_{\mathrm{r}}=\operatorname{diag}\left(\lambda_{1}, \lambda_{2}, \ldots . \lambda_{\mathrm{r}}\right) . \\
& \wedge_{\mathrm{r}}=\left[\begin{array}{ccc}
\lambda_{1} & 0 \\
0 & \lambda_{2 .} & \lambda_{\mathrm{r}}
\end{array}\right]
\end{aligned}
$$

The above is the theory of SVD of the physicist Lanczos $^{[9]}$, so any matrix $\mathrm{G} \in \mathrm{R}^{\mathrm{M} \times \mathrm{N}}$ can be factored as the equation (16), that is $\mathrm{G}$ can be factored as $\mathrm{G}=\mathrm{Ur} \wedge_{\mathrm{r}} \mathrm{V}_{\mathrm{r}}^{\mathrm{T}}$

\section{Let $\mathrm{T}$ :}

$$
\mathrm{G}_{\mathrm{L}}=\mathrm{V}_{\mathrm{r}} \wedge_{\mathrm{r}}^{-1} \mathrm{U}_{\mathrm{r}}
$$

$=\mathrm{V}_{\mathrm{r}} \wedge_{\mathrm{r}} \mathrm{U}_{\mathrm{r}}^{\mathrm{T}} \mathrm{U}_{\mathrm{r}} \wedge_{\mathrm{r}}^{-2} \mathrm{U}_{\mathrm{r}}^{\mathrm{T}}$

$=\mathrm{G}^{\mathrm{T}}\left(\mathrm{U}_{\mathrm{r}} \wedge_{\mathrm{r}}^{-2} \mathrm{U}_{\mathrm{r}}^{\mathrm{T}}\right)^{-1}$

$\mathrm{G}_{\mathrm{L}}=\mathrm{G}^{\mathrm{T}}\left(\mathrm{GG}^{\mathrm{T}}\right)^{-1}$

Be the inverse of the matrix $G, G_{L}$ is called natural inverse by ${ }^{[9]} .{ }^{[10]}$ was named it as Lanczos inverse, and also as generalized inverse and is used based on the above equation for solving linear inversion problems.

So the solution of the $\mathrm{Gm}=\mathrm{d}$ is:

$\mathrm{m}=\mathrm{G}_{\mathrm{L}} \mathrm{d}=\mathrm{V}_{\mathrm{r}} \wedge_{\mathrm{r}}^{-1} \mathrm{U}_{\mathrm{r}}^{\mathrm{T}} \mathrm{d}$

The generalized inversion based on the Lanczos natural inverse, the definition of natural inverse, $\mathrm{m} \mathrm{G}_{\mathrm{L}} \mathrm{d}$ $=\mathrm{r}=\mathrm{N}\langle\mathrm{M}, \mathrm{Gm}=\mathrm{d}$ is an over-determined equation, in this case, the solution of the generalized inversion is:

$\mathrm{m}=\mathrm{G}_{\mathrm{L}} \mathrm{d}=\left(\mathrm{G}^{\mathrm{T}} \mathrm{G}\right)^{-1} \mathrm{G}^{\mathrm{T}} \mathrm{d}$

This is the least square solution which provides the minimum length solution of linear systems. The provided algorithm is based upon the above theory of the generalized inversion.

The application of our algorithm is based on the above steps of processing and was tested on some typical magnetic structures, and on real field data examples using an aeromagnetic data profile over Amenzi area, Inner Mongolia, North China, which targets the shallow igneous body source.

Single anomalous sources: Figure 1-3 represent the total-field magnetic anomalies over single 2-D anomalous source bodies of known depths. By using the structural index of $n=1$, and varying the window size along the profile, the pattern of the clustering around magnetization of geologic interest derives source position and depth. Varying the window size along the profile will show roughly different degree of clustering and also different depths. For a small window, the points give a good tight clustering on the top of the vertical and dipping dike bodies Fig. $(1,3)$ and for horizontal source body the two edges of the source body is indicated by tight clustering points (Fig. 2). 

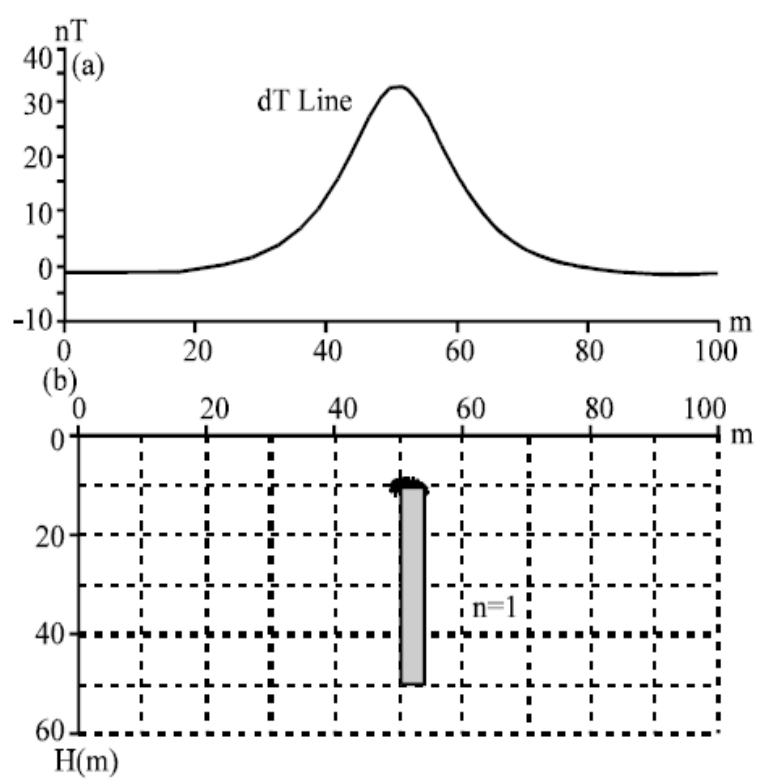

Fig. 1: (a) Shows the Total-field Anomaly (dT) Over Simple Single Anomalous Vertical Dike; (b) The Depths Obtained by Decovolved with Different Window Size and by Using Structural Index $\mathrm{n}=1$ Model 1: Width $=4 \mathrm{~m}$; Height $=$ 40m; Top Depth: 10m; Bottom Depth: 50m; Center Point Coordinate: $(\mathrm{x} 0=50 ; \mathrm{z} 0=12)$
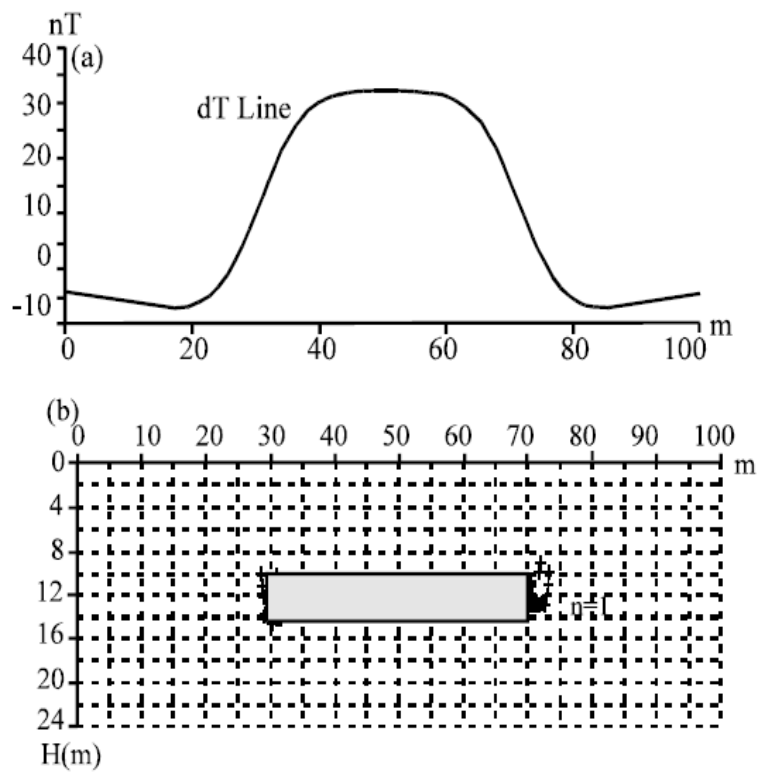

Fig. 2: (a): Shows the Total-field Anomaly (dT) Over Single Anomalous Horizontal Body; (b). The Depths Obtained by Deconvolved with Different Window Size and by Using $\mathrm{n}=1$ Model 2: Width $=40 \mathrm{~m}$; Height $=4 \mathrm{~m}$; Top Depth: 10m; Bottom Depth: 14m; Center Point Coordinate: $(\mathrm{x} 0=50 ; \mathrm{z} 0=12)$
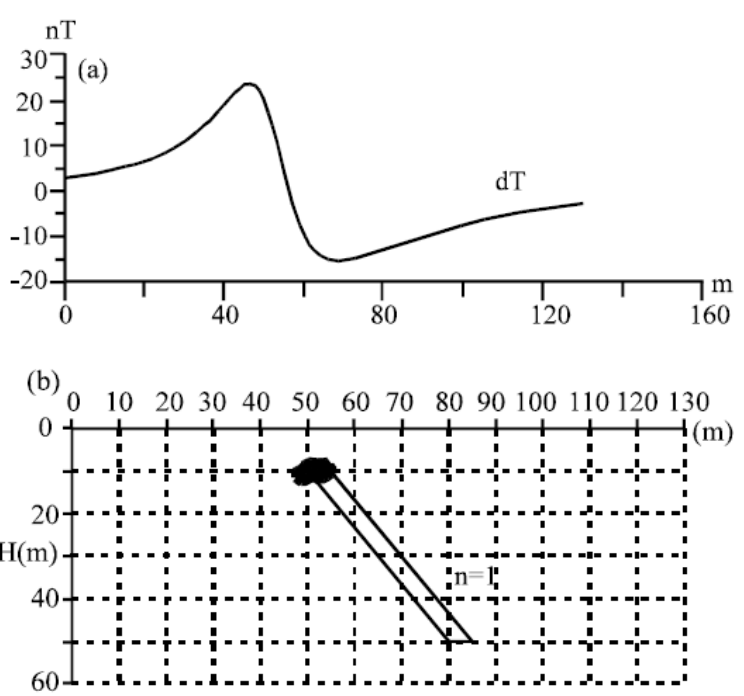

Fig. 3: (a) Shows the total-field Anomaly (dT) Over Single Dipping Anomalous Source Body; (b): The Obtained Depths by Deconvolved with Different Window size and Using Structural Index $n=1$

For a large window, the pattern is represented by only one point on the top of the vertical and dipping bodies and in the case of the horizontal body the pattern is represented by only one point in the center of the source body. In case of horizontal body the geological model may represent the situation where two sedimentary units with different magnetic susceptibilities are juxtaposed which is referred to magnetized contact.

Multiple anomalous sources: In 2-D multiple anomalous sources' case of known depths, the deconvolution was run using different structural indices $\mathrm{n}=0,0.25,0.5$, and 1 and a number of window size along the profile. In case of $n=0$, the program gives poor biased depths, showing that $\mathrm{n}=0$ is a wrong index (Fig. 4a). Using $\mathrm{n}=0.25$ indices, the program gives poor clustering around anomalous bodies and biased depths (Fig. 4b). At $\mathrm{n}=0.5$, the program yields a good solution at the horizontal and dipping bodies, but at the vertical dike gives poor clustering around the geologic body (Fig. 4c). On $\mathrm{n}=1$, and deconvolution with different window sizes, our program yields good clustering and acceptable depths of anomalous bodies varying between $10-14 \mathrm{~m}$ (Fig. $4 \mathrm{~d}$ ).

A real field example from amenzi area: Our algorithm was applied to a real field example of aeromagnetic profile. The aeromagnetic profile has a length of $27 \mathrm{~km}$ located in the southern part of Amenzi area, Inner Mongolia, North China., with a north-south direction. The local anomaly in the south of the profile is interpreted as a shallow-buried igneous body of a depth less than $1000 \mathrm{~m}$, which is corresponding to the positive local magnetic anomaly located in the south part of Amenzi area. 

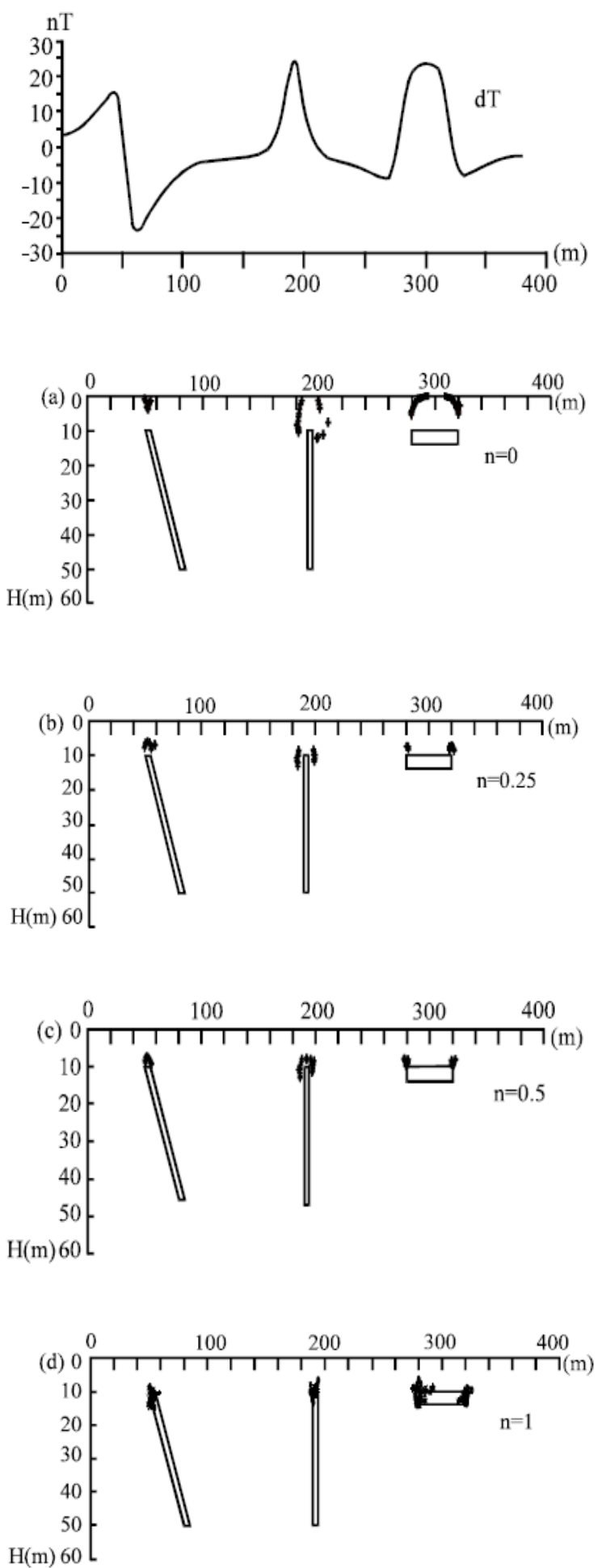

Fig. 4: Shows the Total-field Anomalies (dT) of Multimodel Anomalous Source Bodies and their Depths Deconvolved by Using Different Structural Indices, a) $n=0, b) n=0.25$, c) $n=$ $0.5, \mathrm{~d})$ By Using Correct Index $\mathrm{n}=1$ and Deconvolved by Using Different Moving Window Sizes
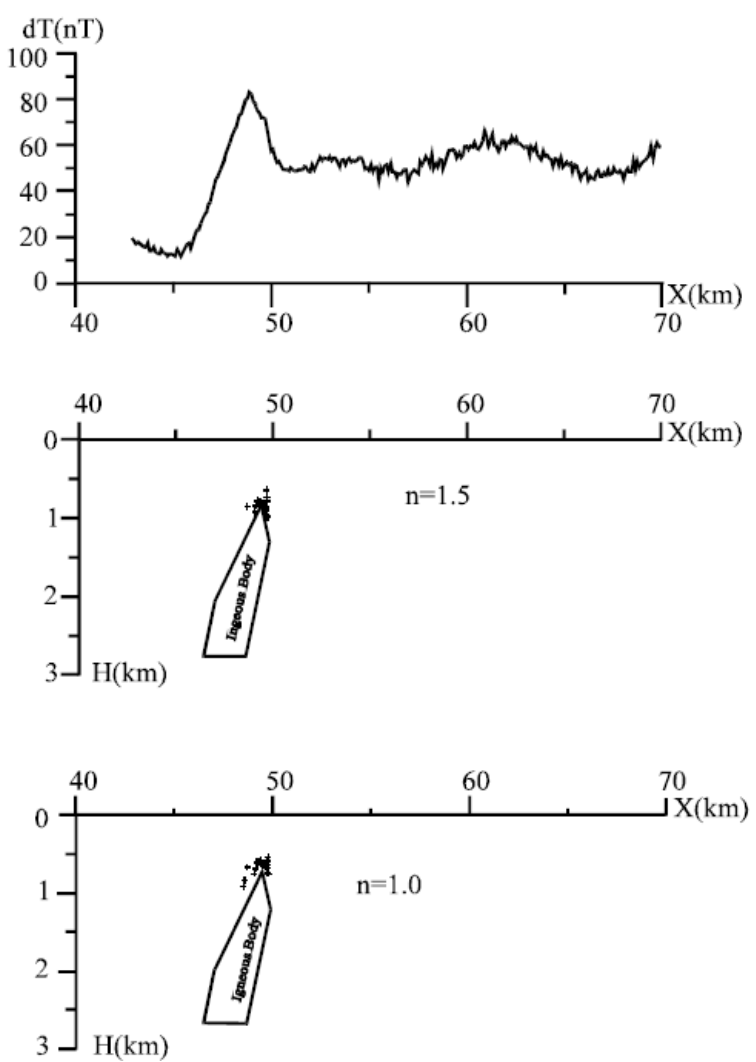

Fig. 5: Shows the Total Magnetic Field (dT) and the Euler Response Over Field Example (Amenzi Area), Using Structural Index $n=1$ and $n=1.5$

The anomalous igneous body was tested by Euler method, using two different structural indices, $\mathrm{n}=1$, and $n=1$. 5. The result was shown in Fig. 5, which shows that the index $n=1.5$ was yielding useful solutions and acceptable depth estimates. The depths vary between $700-900 \mathrm{~m}$.

\section{CONCLUSION}

A number of methods are available for a fast analysis of large data sets. They are used to determine the average location of the main anomalous bodies under a number of quite strict assumptions. Examples include Euler deconvolution methods ${ }^{[1]}$, Werner deconvolution methods ${ }^{[11]}$, the analytic signal approach $^{[12,13]}$ 'statistical' approach and similar methods $^{[14,15]}$.

Euler deconvolution has been widely used in automatic aeromagnetic interpretations because it requires no prior knowledge of the source magnetization direction and assumes no particular interpretation model.

The Euler deconvolution method is best suited for anomalies caused by isolating and multiple anomalous sources. The method is applied to a long profile of aeromagnetic measurements, located over Amenzi area, Inner Mongolia, North China. Some indication of the 
source type can be gained by varying the structural index for any particular feature.

It is concluded from the above the deconvolution must be run with different structural indices even with the intermediate structural indices, which might improve clustering solutions, but they are not related to a known model structure.

The anomalous features of the field example are simple feature and located at shallow depths, so it is better to be deconvolved with a small window size rather than a large window.

\section{REFERENCES}

1. Thompson, D. T., 1982. EULDPH-new technique for making computer-assisted depth estimates from magnetic data. Geophysics, 47: 31-37.

2. Barongo, J. O., 1984. Euler's differential equation and the identification of the magnetic point-pole and point-dipole sources. Geophysics, 49: 15491553.

3. Reid, A.B., J.M. Allsop, H.Granser, A.J. Millett and I.W. Somerton, 1990. Magnetic interpretation in three dimensions using Euler deconvolution. Geophysics, 55: 80-91.

4. Marson, I. and E.E. Klingele, 1993. Advantages of using the vertical gradient of gravity for 3-D interpretation. Geophysics, 58: 1588-1595.

5. Barbosa, V.C.F., B. C. Joao and W.E. Medeiros, 1999. Stability analysis and improvement of structural index estimation in Euler deconvolution. Geophysics, 64: 48-60.
6. Hansen, R.O. and L. Suciu, 2002. Multiple source Euler deconvolution. Geophysics, 67: 525-535.

7. Ravat, D., K. Kirkham and T.G. Hildenbrand, 2002. A source-depth separation filter. Using the Euler method on the derivatives of total intensity magnetic anomaly data. The Leading Edge, 21: 360-365.

8. Penrose, R., 1955. A generalized inverse for matrices. Proc. Cambridge Phil. Soc., 51: 406- 413.

9. Lanczos, C., 1961. Linear differential operators. D. Van Nostrand, Princeton, New Jersey.

10. Jackson, D.D., 1972. Interpretation of inaccurate, insufficient and inconsistent data. Geophys. J. R. Astr. Soc., 28: 97-109.

11. Ku, C. C. and J.A. Sharp, 1983. Werner deconvolution for automated magnetic interpretation and its refinement using Marquardt's inverse modeling. Geophysics, 48: 754-74.

12. Roest, W.R., J. Verhoef and M. Pilkington, 1992. Magnetic interpretation using the 3-D analytic signal. Geophysics, 57: 116-125.

13. Spector, A. and F. S. Grant, 1970. Statistical models for interpreting aeromagnetic data. Geophysics, 35: 293-302.

14. Blakely, R.J. and R.W. Simpson, 1986. Approximating edges of such bodies from magnetic or gravity anomalies. Geophysics, 51: 1494-1498.

15. Miller, H.G. and V. Singh, 1994. Semiquantitative techniques for the identification and removal of directional trends in potential field data. J. Appl. Geophys., 32: 199-211. 